LBNL-51413

\title{
INDOOR POLLUTANT MIXING TIME IN AN ISOTHERMAL CLOSED ROOM: AN INVESTIGATION USING CFD
}

\author{
A.J. Gadgil ${ }^{1}$, C. Lobscheid ${ }^{1,2}$ and M.O. Abadie $^{1}$ \\ ${ }^{1}$ Indoor Environment Dept., Lawrence Berkeley National Laboratory, Berkeley, CA \\ 94702, USA \\ ${ }^{2}$ Technical University of Berlin, Berlin, Germany \\ e-mails: ajgadgil@,lbl.gov, mabadie@univ-lr.fr
}




\begin{abstract}
We report computational fluid dynamics (CFD) predictions of mixing time of a point pulse release of a pollutant in an unventilated mechanically mixed isothermal room. The aims of the study are to determine (1) the adequacy of the standard RANS two-equation $(\mathrm{k}-\varepsilon)$ turbulence model to predict the mixing times under these conditions, and (2) the extent to which the mixing time is a feature of the room airflow, rather than the source location within the room. CFD simulations modeled the twelve mixing time experiments performed by Drescher et al. (1995) in an isothermal sealed room for a point pulse release. Predictions of mixing time were found in good agreement with experimental measurements, over an order of magnitude variation in blower power. Additional CFD simulations were performed to investigate the relation between pollutant mixing time and pollutant source location. Seventeen source locations were investigated for five different blower power configurations in the room. Results clearly show large dependence of the mixing time on the room airflow, with some dependence on source location. We further explore dependence of mixing time on the local airflow properties (velocity and turbulence intensity) at the source location. Implications for our findings for positioning air-toxic sensors in rooms are also discussed.
\end{abstract}

Keywords: mixing time, pollutant dispersion, CFD modeling, short-term exposures 


\section{Introduction}

Indoor air quality investigations usually assume a uniform distribution of pollutants throughout the interior space. This assumption of instantaneous mixing offers some compelling advantages in domain of its validity. For experimental purposes, the measurement of the concentration at only one point can be used to obtain the overall concentration throughout the room. In modeling studies, the assumption leads to governing equations that are either systems of ordinary differential equations or algebraic equations, whose numerical solutions are straightforward in contrast to the partial differential equations that must be solved if one takes real mixing into account.

However, particularly for point sources, and for short-term or localized indoor exposures, this assumption proves too simplistic for the initial period of the mixing of the pollutant in the room air. Lambert et al. (1993) showed, for example, that the levels of respirable suspended particles and nicotine were respectively $40 \%$ and $65 \%$ lower in no-smoking sections of restaurants than in the smoking sections. The mixing problem has two aspects: (1) how to determine the conditions under which the well-mixed approximation is inappropriate, and (2) how to model pollutant concentrations when the well-mixed approximation is inappropriate. We earlier addressed the first question experimentally for rooms mixed with natural or forced convection (Baughman et al. 1994, Drescher et al. 1995). A "mixing time" is defined for a point pulse release of a pollutant in the room, under particular flow conditions. At times beyond the mixing time one can safely use the well-mixed assumption. At times earlier than the mixing time, the well-mixed assumption is a poor approximation. 
Some of the previous work on modeling imperfectly mixed pollutant concentrations aimed for the introduction of an additional mixing factor (Hoegg, 1972) (Ishizu, 1980). More recently, a two-compartment model was applied, artificially defining a small virtual space around the source (e.g., an area of workplace pollutant emissions) also with a uniform concentration, but different from that in the rest of the room (Furtaw, 1996). Although this construction allowed for initial concentration build-up near the source, comparisons with experiments showed that concentrations near the source exceeded the predictions of this modeling approach. In the current work, we focus not on improving models of exposure under poorly mixed conditions, but on numerical investigation of mixing time itself: Is it possible to predict it numerically using the standard ( $k-\varepsilon)$ turbulence model, does the mixing time depend on source-location and if so to what extent, and finally, are there any implications of our findings for recommending locations of sensors for acutely toxic pollutants in a room.

Computational fluid dynamics (CFD) solves the partial differential equations governing air motion and pollutant mixing and can provide solutions with high spatial and temporal resolution. With continuing improvements in CFD methods and increased availability of computer power, CFD is an increasingly common tool in research. Nevertheless, making accurate CFD predictions of room airflow remains a demanding enterprise, requiring experience and care in defining the grid, treatment of boundary conditions, and numerical properties of the model. 
Most of the previous work on the CFD simulation of contaminant dispersion in a room was performed without corresponding experiments (Baker and Kelso, 1990). Yaghoubi et al. (1995) investigated mixing of pollutants using simulations with varied emissions from a pollutant source, cooling and heating locations, and incoming air temperatures. Roy et al. (1994) used a CFD code to predict contaminant dispersion in a kitchen-hood geometry. Gadgil et al. (2000) provide a brief review of experimental and computational research investigations of pollutant dispersion in indoor spaces.

The two specific goals of this research were to investigate (1) the adequacy of the standard $(k-\varepsilon)$ turbulence model for predicting the mixing time of a point-source release, and (2) the extent to which the mixing time for a given room airflow is a characteristic of the airflow, rather than the release location of the pollutant. For reference we used results from previous experiments releasing a point pulse of Carbon Monoxide (CO) in an isothermal unventilated room with forced convection airflow. We simulated a series of 12 such experiments spanning about one order of magnitude in the power output from the blowers in the room. Additional simulations were performed to study the relation between pollutant mixing time and pollutant source location.

\section{Mixing time definition}

Given a point pulse release of a pollutant in a room with no exhaust or fresh air supply, the pollutant will be eventually uniformly distributed throughout the room. If we denote the time elapsed after the point pulse release as $t$, then a characteristic mixing time, $\tau_{m i x}$, can be defined such that for $t$ smaller than $\tau_{m i x}$, the pollutant concentration varies 
substantially throughout the room, and for $t$ larger than $\tau_{m i x}$, the pollutant concentration is essentially uniform throughout the room. The mixing time is defined as the interval from the time of point-pulse release to the time at which the standard deviation of the pollutant concentration $C_{i}(t)$ (measured at $N$ monitoring points in the room) drops permanently below $10 \%$ of the arithmetic mean concentration $\bar{C}(t)$ of the pollutant at these monitoring points. $N$ must be sufficiently large and the monitoring points sufficiently dispersed in the room that additional monitoring points do not change the value obtained for the mixing time.

$$
\text { At } \quad t=\tau_{\text {mix }} \quad \frac{1}{\bar{C}(t)} \sqrt{\frac{\sum_{i=1}^{N}\left(C_{i}(t)-\bar{C}(t)\right)^{2}}{N}}=0.10
$$

\section{Experiment Description}

Figure 1 presents isometric and plan views of the experimental room, which measured $3.53 \mathrm{~m} \times 3.74 \mathrm{~m} \times 2.36 \mathrm{~m}$ high $\left(31 \mathrm{~m}^{3}\right)$. Vertical edges of the room are denoted in the isometric and plan views for later discussion. Five independently powered identical centrifugal fans, each fitted with a $60 \mathrm{~cm}$ long plastic exhaust pipe, were placed $3 \mathrm{~cm}$ above the ground with their plastic exhaust pipes parallel to one another and to the floor. In each experiment, after the room airflow was established, 1.5 liter of pure CO was released into the unoccupied room over a period of 20 seconds through a perforated cylinder. The $\mathrm{CO}$ concentrations at various locations in the room were monitored with nine sensors. Each experiment was replicated once. Temperatures at several points in the room were recorded. Attempt was made to reach isothermal conditions in the room - 
such conditions were not attained exactly and experimenters applied a correction to remove the influence of natural convection from experimentally measured mixing time (they report both uncorrected and corrected values of mixing times). Detailed experimental descriptions can be found in Drescher (1994) and Drescher et al. (1995). It is important to note that the range of blower power (and blower exhaust velocities) spanned in the experiment was within the realistic values encountered in the indoor environment.

\section{CFD modeling}

\subsection{Grid and boundary conditions}

Most of the room space was discretized with a coarse $10 \mathrm{~cm} \mathrm{X} 10 \mathrm{~cm} \mathrm{X} 10 \mathrm{~cm}$ mesh, except near the room boundaries and near the blower exhausts, where the grid was appropriately refined. An inner large block of cells surrounding and enclosing the blowers was aligned with the exhaust pipes of the blowers to avoid imposing the grid orientation on the exhaust jets. The blowers and attached pipes were simulated using block structures with $5 \mathrm{~mm}$ resolution at the ends of the blower pipes where the highest velocities were expected. The space inside the blowers and the exhaust pipes was excluded from the computational domain. The walls of the room and the outside surfaces of the blowers and pipes were defined as impermeable non-slip boundaries. The blower inlets and exhausts were defined to have prescribed flows with fixed velocity profiles. User subroutines were implemented so that the pollutant concentration entering the blower inlet would appear at the blower outlet at the next time step. 
Grid independence of the CFD solution is necessary (but not sufficient) for good prediction. We recorded the steady-state velocities at a number of locations where the velocities were large or had large gradients (e.g., near the blower exhausts). The grids were successively refined until no significant changes in these velocities resulted. The final working grid was chosen to be the one from the penultimate of the successive refinement steps. This approach does carry a risk of missing important areas or location where further refinement would have made a measurable difference in the concentration field, hence the monitoring locations need to be carefully selected. Figure 2 presents the final working grid of 130,942 nodes.

Predictions of transient pollutant dispersion must be independent of the time-step. We used a time step of 0.1 second for the simulation of pollutant dispersion in the "basecase" (all five blowers operating at an average exhaust air velocity of $1.86 \mathrm{~m} / \mathrm{s}$ ). Then we tested the predictions with those obtained using a 0.5 second and a 0.05 second time steps. The mixing time predicted with the small time step ( $0.05 \mathrm{~s})$ was 198 seconds, and for the large time step $(0.5 \mathrm{~s}) 196$ seconds. These compared well with the predicted mixing time of 197 seconds of the simulation using 0.1 second time step, and this timestep was retained for the other simulation predictions.

\subsection{Computational Procedure}

The Navier-Stokes equations were solved with a commercial code based on a finitevolume fully implicit method. We selected the standard (high Reynolds number) Reynolds Averaged Navier Stokes (RANS) two-equation $(k-\varepsilon)$ model for turbulence. In 
an earlier separate investigation, we determined that predictions using first order upwind differencing scheme gave unsatisfactory agreement with experimental observations; we found much improved agreement using the second-order MARS (Monotone Advection and Reconstruction Scheme) method. In the current investigation, only MARS scheme was employed for both velocities and pollutant transport modeling. The SIMPLE (Semi Implicit Method For Pressure Linked Equations) algorithm was applied for the calculations of the steady-state velocity fields (without pollutant) and PISO (Pressure Implicit Split Operator) for the transient calculations of the pollutant dispersion in the room (with pre-calculated steady-state velocities and turbulence parameters).

The chosen convergence criterion was that the residuals for all calculated dependent variables decreased at each cell below a value of $10^{-4}$ for the steady state calculations. Numbers of iterations to reach convergence for the steady state ranged from 1817 to 4855, requiring 10 to 35 hours processing time on a Sun Origin 2000 server with $2 \mathrm{~GB}$ RAM and two processors. The transient calculations of pollutant dispersion (with the precalculated velocity field) required about half as much processing time.

Details of grid generation and testing, time-step control, and computational procedure are described in Lobscheid (2001).

\section{Comparison of CFD results with experiments}

In the experiments, temperatures at the walls and at different heights in the middle of the room differed from the average temperature by amounts ranging from 0.25 to 1.0 Kelvin 
with an average of 0.59 Kelvin. The influence of natural convection was neglected in the CFD simulations. Predictions are thus compared to the corrected mixing times reported in Drescher et al (1995).

Figure 3 compares the pollutant mixing time predicted with CFD and those reported from experiments. Extremities of horizontal bars in Figure 3 show the experimentally measured values in replicate experiments. As can be seen, there's some variability in the experimental results and measured values of replicate experiments differ on average by $18 \%$. In comparison, the experimentally measured mixing times span about one order of magnitude (from 2.4 to 23 minutes)

The dashed line in Figure 3 indicates a perfect fit. Most of the datapoints in Figure 3 lie below this line -- the CFD calculations underestimate mixing times by an average of $30 \%$. One possible reason for this general underprediction is that the flow fields in the room were not everywhere turbulent. Especially in the cases with low blower power resulting in low air velocities (i.e. high mixing time), parts of the room were probably laminar, e.g., in the middle of the room near the stagnation center of the bulk flow. Therefore a low-Reynolds number k- $\varepsilon$ model might be better at predicting the mixing times at the low end of the blower power range (i.e., at the high end of the mixing time range). As Heiselberg (1996) concluded, comparisons between measurements and CFD simulations at low air change rates will show large deviations if the numerical simulation of the air and contaminant distribution does not take the low-Reynolds-number effects into consideration. Another explanation of the mixing time discrepancy lies in the limited 
number of experiments; only two experiments were performed for each configuration of blower power, and thus the experimental data are inherently noisy.

\section{Impact of the pollutant source location on the mixing time}

Additional simulations were performed to study the dependence of the mixing time on the location of the pollutant release. Five airflow configurations were chosen from the previous set of experiments to span the range of blower power (i.e., of average air velocity). In all configurations, the physical positions of the blowers remain fixed, only the power supplied to various blowers (in combination) is different. For this reason, it is instructive to briefly describe the major features of the airflow in the room.

The exhaust jets from the blower outlets run parallel to the floor, rapidly merging with one another, and entraining the room air as they move toward the East wall of the room. At the East wall, the jets cause a flow to climb up the East wall towards the ceiling. This airflow continues to entrain more room air and slows down. It creates a large loop of air in the vertical plane of the blowers that is kept going by the main exhaust jets. This loop has a center, about 1.0 meter above the blowers with very little air motion. Secondary recirculation loops are created as some of the air from the jets moves towards the South and North walls on reaching the East wall. A small stable loop is created in the lower SE corner that has relatively low air exchange with the main vertical loop. Overall, the air velocities are high in the lower East edge of the room, and along the floor in the path of the jets. Velocities are much lower in the lower SW corner. 
Seventeen pollutant source locations were investigated. The locations were selected considering two criteria:

- The locations should be possible points for a Chem/Bio agent release in the room. This suggested investigating those locations where letters or boxes may be stored or opened (location height $<1.5 \mathrm{~m}$ ), and

- The set of locations should obtain the broad range of mixing time values. Consequently, some sources have to be located close to the blowers zone where the pollutant dispersion would be rapid (lower limit of mixing time range) and others away from this zone, in quiescent regions of the room (upper limit of mixing time range).

Figure 4 presents the seventeen pollutant source locations: twelve are located "on" the floor (in the 4 floor corners, at the centers of the 4 edges of floor, and 4 in the central region of the floor, more than a meter from any wall) and five are located 0.75 meter high (in the core region of the room airflow, more than a meter from any wall). Each source has volume one liter, roughly cubical, about $10 \mathrm{~cm}$ to the side. The sources on the floor have one surface of the cube touching the floor, but rest of the source volume extends about $10 \mathrm{~cm}$ vertically, outside the boundary layer lining the floor

A grid of 48 sensors evenly distributed within the room volume was used to record the concentration as the pollutant dispersed in the room during the simulation. The larger number of sensors was chosen to obtain a better evaluation of the pollutant heterogeneity within the room volume. Every sensor is representative of a supposedly well mixed 0.6 
$\mathrm{m}^{3}$ region in the room centered on the sensor (instead of $3.4 \mathrm{~m}^{3}$ that must be represented by each of the 9 sensors). This thus provides us with a more accurate measure of mixing time.

Figure 5 presents in four graphs the pollutant mixing time for various source locations plotted against the average air speed in the room. Predicted mixing times are separately plotted for sources in corners, floor edges, in the center region of the floor, and in the core of the room. On each graph, we also plot the predicted mixing times for the release location used in the Drescher et al (1995) experiments already shown in Figure 4 (hollow diamonds). These are provided as reference data points for ease of comparison across graphs.

The one feature that stands out in Figure 5 is that the mixing times are not out of the trend lines in any of the four graphs, compared to the mixing times for the experimental release location (points indicated with diamonds). Irrespective of release location, when the average air speeds in the room are high, the mixing times are short. When the average room air speeds are low, the mixing times are long. This variability in the mixing time attributable to the differences in the average room air speed is about a factor of 10 . The scatter (or variability) arising from source location in the predicted the mixing time is indicated by the vertical height of the band of points in the graphs. Across the whole range of air speeds, this remains about a factor of 2 . 
Further examination of specific mixing times for various locations show, as expected, that the mixing times are longer for pollutant sources located in the corners and at the edges of the room compared to the source locations in the room core or on the central part of the floor. Two different ranges of mixing time are identifiable, one for the sources located in the corners and at the edges and another for those located on the floor and in the core region. The difference between the ranges is especially prominent for low air speed. For a given average room air speed, the mixing time for release locations in the room's core volume are the shortest, and those from the corner and edges are the longest (within the factor 2 range).

One significantly large mixing time value is observed for each air speed, and this is obtained with the pollutant release in the SE corner of the floor (Figure 4). Visualization of air velocities the pollutant concentrations from the transient simulation provides an explanation. The pollutant released in this particular corner tends to stay in the source region because of an air circulation loop located in this corner. This closed loop causes the pollutant to circulate in this corner and disperse slowly into the room compared to the releases at other locations, giving rise to the longest mixing time for any release location. It is remarkable that this mixing time consistently exceeds even that for the pollutant release in the floor corner with the lowest local air speed (the SW corner). 


\section{Mixing time versus local air characteristics at source}

In the previous section, we explored the dominant effect of average room air speed on the pollutant mixing time. For a given air speed, there is smaller, but still significant effect of the source location on the mixing time.

As previously mentioned, the mixing time becomes longer for pollutant sources located far from the main air flow, i.e. the blowers and the main vertical air recirculation loop driven by the blower jets. We can separate the source locations into 2 groups: the sources located near the vertical walls (corners and edges) and those in the core region. Mixing times for the latter group are in the lower half of the range and those for the first group are in the upper half. It's more difficult to make further distinctions within each group of locations. In general, once the pollutant reaches either the inlet region of the blowers, or gets entrained in the jets coming out of the blowers, rapid mixing thereafter is assured. Determining the time for the pollutant to reach the blowers zone by air transport is feasible with detailed knowledge of the three dimensional air flow patterns. However, this approach does not yield useful insights generalizable to practical situations for other room geometries and other air supply configurations.

We take another approach in this section -- to investigate whether the local flow characteristics at the source are useful predictors of mixing time. The pollutant dispersion away from the source release point is caused by advection (the air velocity), and diffusion (dominated by air turbulence) at the source. If advection and dispersion are locally strong, the pollutant will quickly disperse away from the source and perhaps enter 
the general circulation in the room. Figure 6 and 7 respectively present pollutant mixing times plotted against the local air velocity and local turbulence intensity at the source location. The five different blower power configurations are distinguished from one other in the figures by the average room air velocities in Figure 6 and by the average turbulent intensity in Figure 7.

Turbulent intensity is defined by:

$$
I=\frac{\sqrt{\frac{2}{3} k}}{U}
$$

where $I$ is the turbulent intensity (-), $k$ is the turbulent kinetic energy $\left(\mathrm{m}^{2} / \mathrm{s}^{2}\right)$ and $U$ is the velocity magnitude $(\mathrm{m} / \mathrm{s})$.

Figure 6 shows that the pollutant mixing time is short if air velocity at the source is higher than about $0.2 \mathrm{~m} / \mathrm{s}$. This applies, for example, for source locations in the path of the exhaust jets from the blower. However, if the air velocity is lower than $0.2 \mathrm{~m} / \mathrm{s}$, mixing time values are scattered - the mixing times can be long or short depending on the local flow details. For example, the region upstream of the blower inlets has relatively low velocities, but the mixing time for releases from that region are short (owing to entrainment into the blower intake flow and subsequent high speed ejection from the exhaust). On the other hand, air speeds are relatively low in other corners and produce long mixing times. 
Figure 7 shows mixing time plotted against turbulence intensity at the source. The points are widely scattered, and it is difficult to draw generalized trends from the figure. Taken together, Figures 6 and 7 suggest that locally high air speed is a better predictor of short mixing time than locally high value of turbulent kinetic energy. In other words, advection, rather than turbulent diffusion, is the locally dominant mechanism in situations with short mixing time. This makes sense given that real rooms (as also the one modeled in this paper), unlike stirred tank chemical reactors, have low turbulent intensities, and therefore low turbulent diffusivities. We discuss in the next section implications of this for positioning indoor sensors for detecting toxic airborne threats.

\section{Discussion and Conclusion}

In this study, we found that the standard $(k-\varepsilon)$ turbulence model, using a carefully generated grid, boundary conditions and the MARS solution algorithm, suppressing numerical diffusion, gives mixing time predictions within $30 \%$ of experimental measurements over an order of magnitude range in mixing time.

We explored the mixing times for 17 different release locations for five settings of blower power spanning realistic range of mechanical power supplied to room air in real buildings. Pollutant mixing time was found to primarily depend on the mean airflow in the room, and secondarily on the pollutant source location. For identical airflow characteristics, mixing time values for sources located near the walls (in the corners and at the edges of the room) are about twice higher than those located in the core region. 
Predictions of mixing time can be made based on the local air velocity at the source location only for high air velocity (greater than about $0.2 \mathrm{~m} / \mathrm{s}$ ). Turbulent intensity at the source location is poorly correlated to the pollutant mixing time.

It is possible to ask the inverse question. Instead of estimating the mixing time from localized pollutant releases as described above, what are the implications of these findings for best positions for sensors in a room. In the discussion below, we use as shorthand "proximate downwind" and "distant downwind" to describe sensor locations in the room that experience respectively short and long delays for an airborne toxic in the room to reach them. We also use "proximate upwind" and "distant upwind" as shorthand to describe locations of sources that quickly or slowly disperse into the room air.

For a given airflow, there is a parallel in the desired placement of a sensor that should quickly detect a highly toxic airborne release, and the placement of a source that has a short mixing time. The parallel arises from two considerations. First, consider the dominant mechanism of advective transport. In a steady-state incompressible airflow in a closed (i.e., unventilated) room, there are no points that are locally "downwind" or "upwind" of the rest of the room. Any source that has a short mixing time, and its location is thus proximate upwind of the room airflow, is also positioned proximate downwind of the room airflow (since some room air must come to replace the air that leaves the source location). A source that has a long mixing time, and is thus located distant upwind of the general room airflow, can be only distant downwind of the general 
room airflow. This would apply, for example, for a source located inside a closet in the room with a low air exchange with the rest of the room. Second, consider the less significant transport by turbulent diffusion. Here the argument is straight forward. Diffusion is always two way process; if high diffusivity permits rapid source dispersion down the concentration gradient from the source to the room, it will also allow rapid access for the sensor to any airborne toxic in room, down the gradient from the room to the sensor. A source location that permits poor dispersion owing low turbulent diffusion, will also correspondingly be an unattractive location for a sensor. As an example, the SE corner of the floor discussed in section 6 sits in a closed recirculation loop and a source located in this corner has relatively long mixing time. One can thus expect that a sensor located in this corner will also need a long time to detect a pollutant released in the room air.

This argument of course needs to be modified for real rooms that have air supply and air exhausts. A pollutant released near the air exhaust may not mix in the room at all, and a sensor located at the fresh air inlet may never detect a toxic substance released in the room. Exchange of air from the room to the outside destroys the symmetry between source and sensor locations. Further investigation is needed explore generalizable principles for such situations. 


\section{Acknowledgements}

The authors are grateful to Elizabeth Finlayson for many helpful suggestions and much advice during the computational work with the commercial software code. This work was supported by the Office of Nonproliferation Research and Engineering, Chemical and Biological National Security Program, of the National Nuclear Security Administration under U.S. Department of Energy Contract No. DE-AC03-76SF00098.

\section{References}

BAKER, A.J., and KELSO, M. (1990) "On validation of computational fluid dynamics procedures for room air motion prediction", ASHRAE Transactions, 96(1), 760-774.

BAUGHMAN, A.V., GADGIL, A.J., and NAZAROFF, W.W. (1994) "Mixing of a point source pollutant by natural convection flow within a room", Indoor Air, 4, 114-122.

DRESCHER, A.C. (1994) Computed Tomography and Optical Remote Sensing: Development for the Study of Indoor Air Pollutant Transport and Dispersion, Ph.D. Thesis, Dept. of Civil and Environmental Engineering, University of California, Berkeley.

DRESCHER, A.C., LOBASCIO, C., GADGIL, A.J., et al. (1995) "Mixing of a point source indoor pollutant by forced convection", Indoor Air, 5, 204-214.

FURTAW, J., PANDIAN, M.D., NELSON, D.R., et al. (1996) "Modeling indoor air concentrations near emission sources in imperfectly mixed rooms", Journal of Air and Waste Management Association, 46, 861-868. 
GADGIL, A.J., FINLAYSON, E.U., FISCHER, M.L., et al. (2000) "Pollutant transport and dispersion in large indoor spaces: a status report for the large space effort of the interiors project", Lawrence Berkeley National Laboratory Report, LBNL-44791, Berkeley, USA.

HEISELBERG, P. (1996) "Room air and contaminant distribution in mixing ventilation", ASHRAE Transactions: Symposia, 332-339.

HOEGG, U.R. (1972) "Cigarette smoke in closed space", Environmental Health Perspectives, 2, 117-128.

ISHIZU, Y. (1980) "A general equation for the estimation of indoor pollution", Environmental Science \& Technology, 14, 1254-1257.

LAMBERT, W.E., SAMET, J.M. and SPENGLER, J.D. (1993) "Environmental tobacco smoke concentrations in no-smoking sections of restaurants", American Journal of Public Health, 83, 1339-1341.

LOBSCHEID, C. (2001) Isothermal mixing of a point source indoor pollutant: numerical predictions and comparison with experiments, Diploma Thesis, Faculty of Process Engineering, Hermann-Rietschel Institute, Technical University of Berlin, Germany. ROY, S., KELSO, R.M., and BAKER, A.J. (1994) “An efficient CFD algorithm for the prediction of contaminant dispersion in room air motion", ASHRAE Transactions, 100(2), 980-987.

YAGHOUBI, M.A., KNAPPMILLER, K.D., and KIRKPATRICK, A.T. (1995) “Threedimensional numerical simulation of air contamination dispersal in a room", ASHRAE Transactions, 101(2), 1031-1040. 


\section{Figures Captions}

Figure 1. Schematic of the experimental room (isometric and plan view)

Figure 2. Final computational grid in the plan view

Figure 3. Comparison between experimental measurements and numerically predicted mixing times. Each experiment was replicated once, and both the experimental measurements are shown.

Figure 4. Seventeen simulated pollutant source locations, and the grid of 48 sensors, shown in plan view. The sensors, labeled "S", are located three stacks of 16 in each horizontal plane. In the plan view only one such stack can be seen since the other two are coincident with the first.

Figure 5. Mixing time for various groups of source locations is plotted against average room air speed in the figure. Note the vertical axis is logarithmic. This, roughly uniform vertical width of the band of points translates into a roughly uniform multiplicative factor for the range of points for a given value of average room air speed.

Figure 6. Mixing time for various source locations plotted against the magnitude of the local air velocity. The five different symbols represent five different simulations, each with a different blower power. These are keyed in the figure caption box by the average room air velocity, which spans an order of magnitude. Whenever the local air velocity exceeds about $0.2 \mathrm{~m} / \mathrm{sec}$, the mixing time is invariably short, presumably because the pollutant is quickly transported into other parts of the room, at least some of which will permit rapid dispersion. For lower values of local air velocity, the mixing times range widely, from very short to very long. See text for explanation. 
Figure 7. The mixing time plotted against air turbulent intensity at the pollutant source location. The five different symbols represent five different simulations, each with a different blower power. These are keyed in the figure caption box by the average room air velocity, which spans an order of magnitude. 

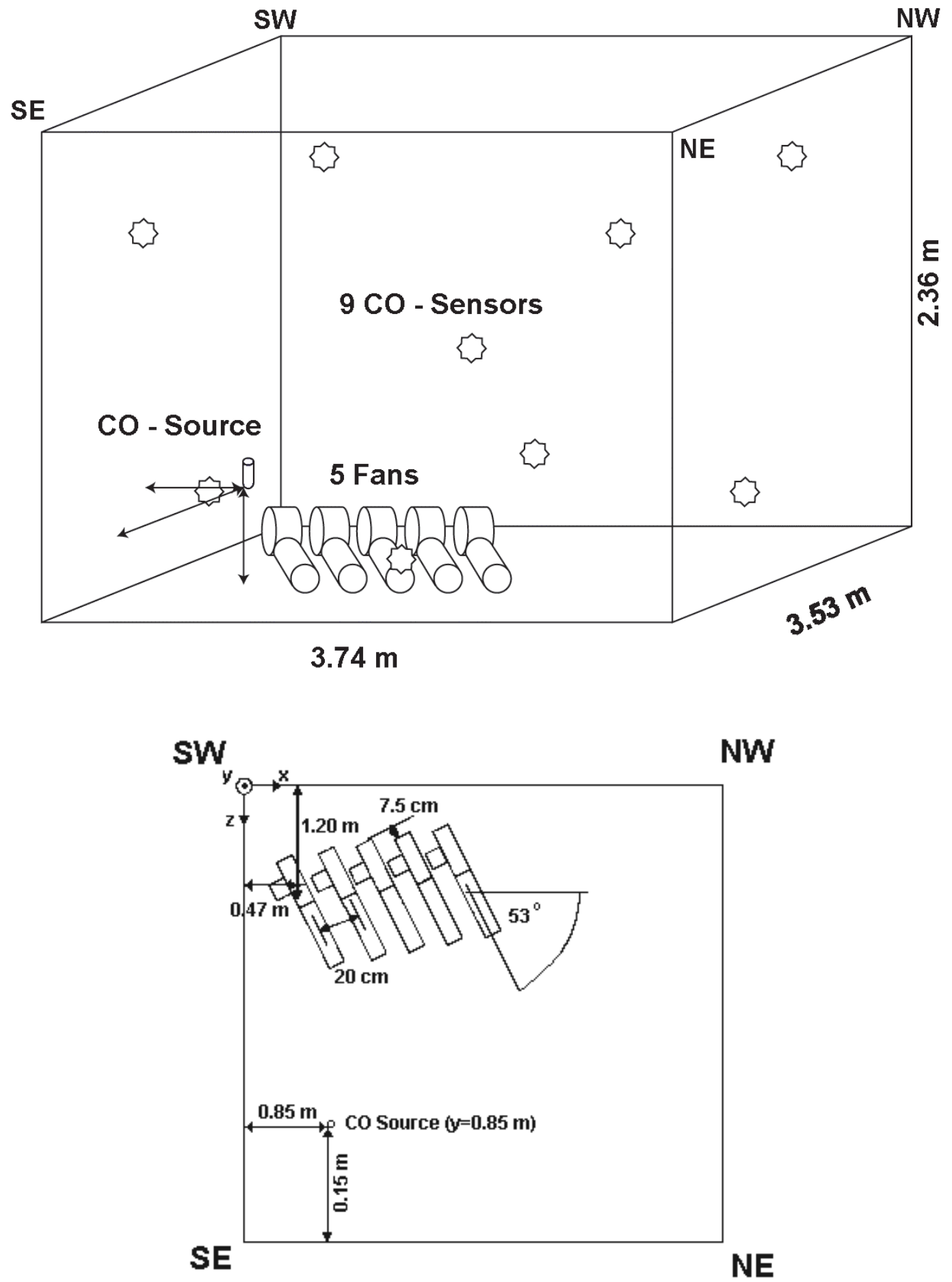

Figure 1 


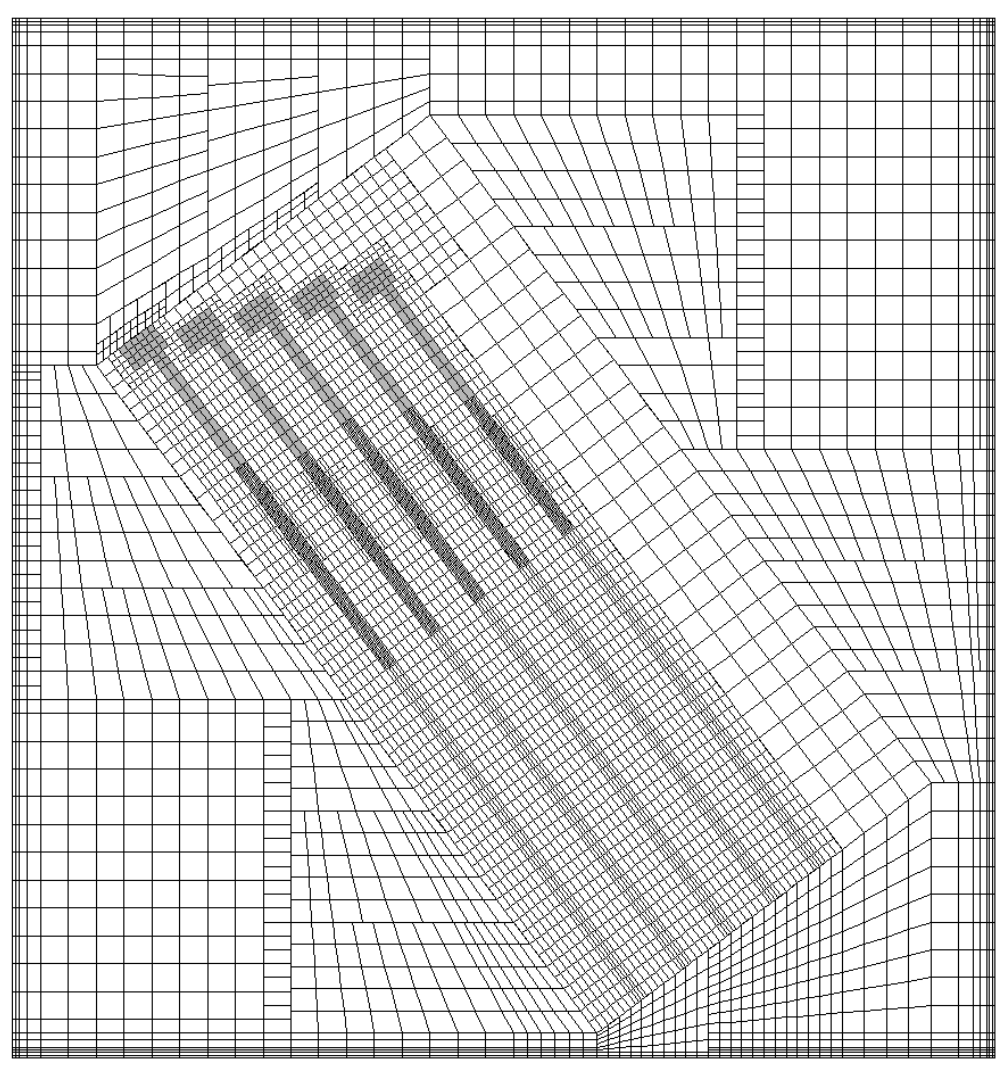

Figure 2 


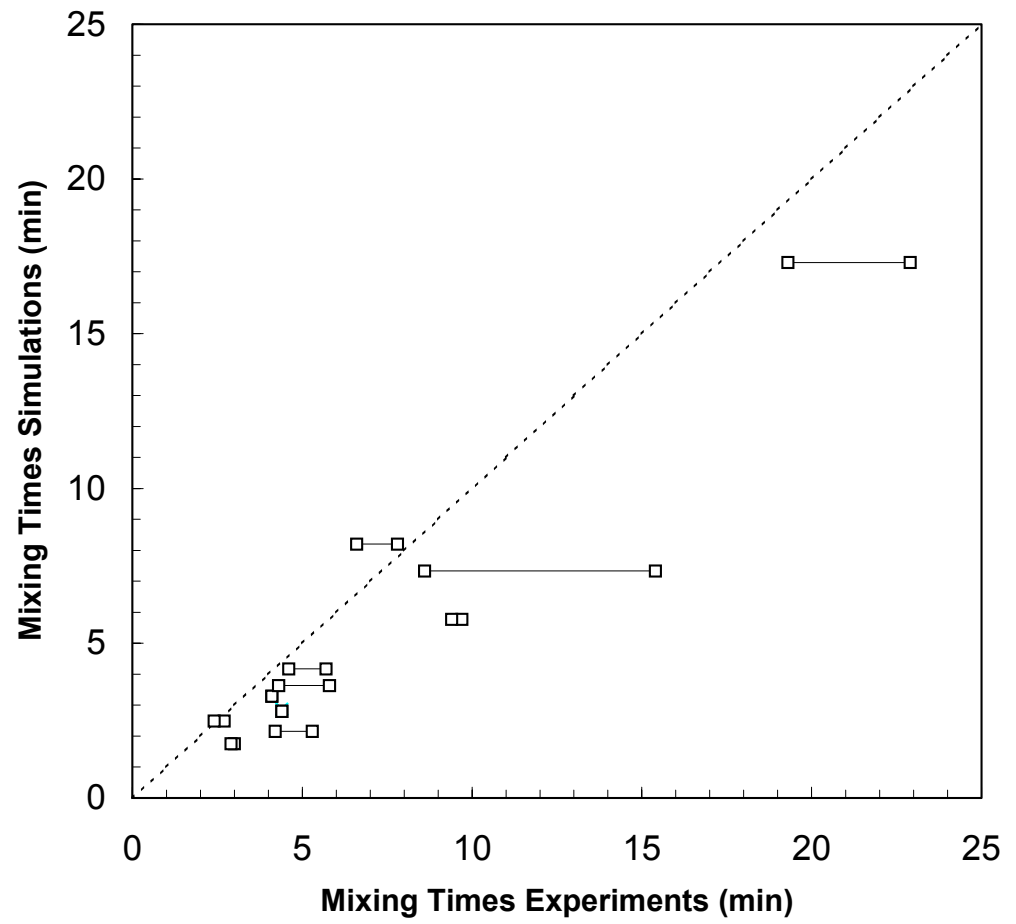

Figure 3 


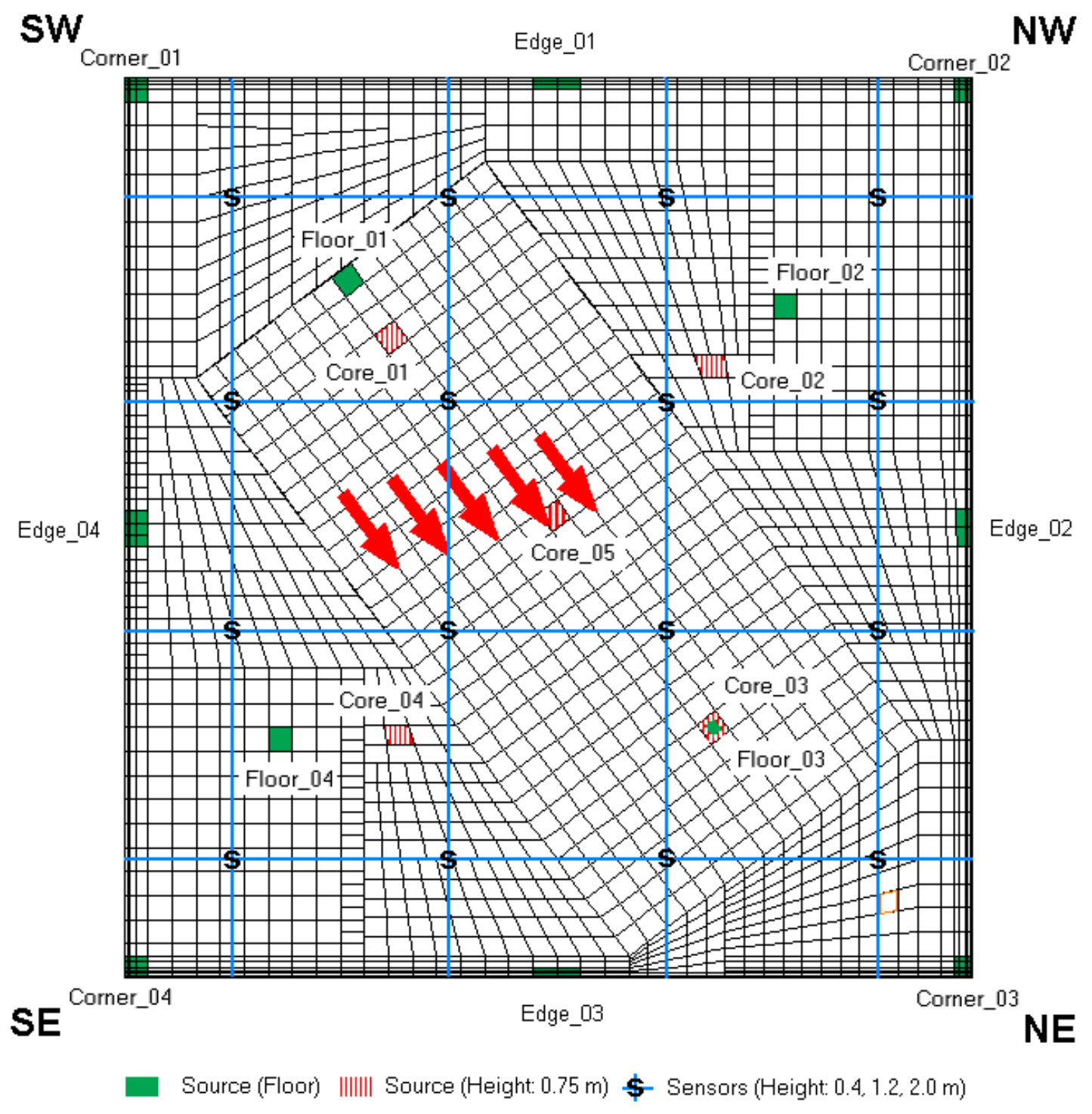

\section{Figure 4}



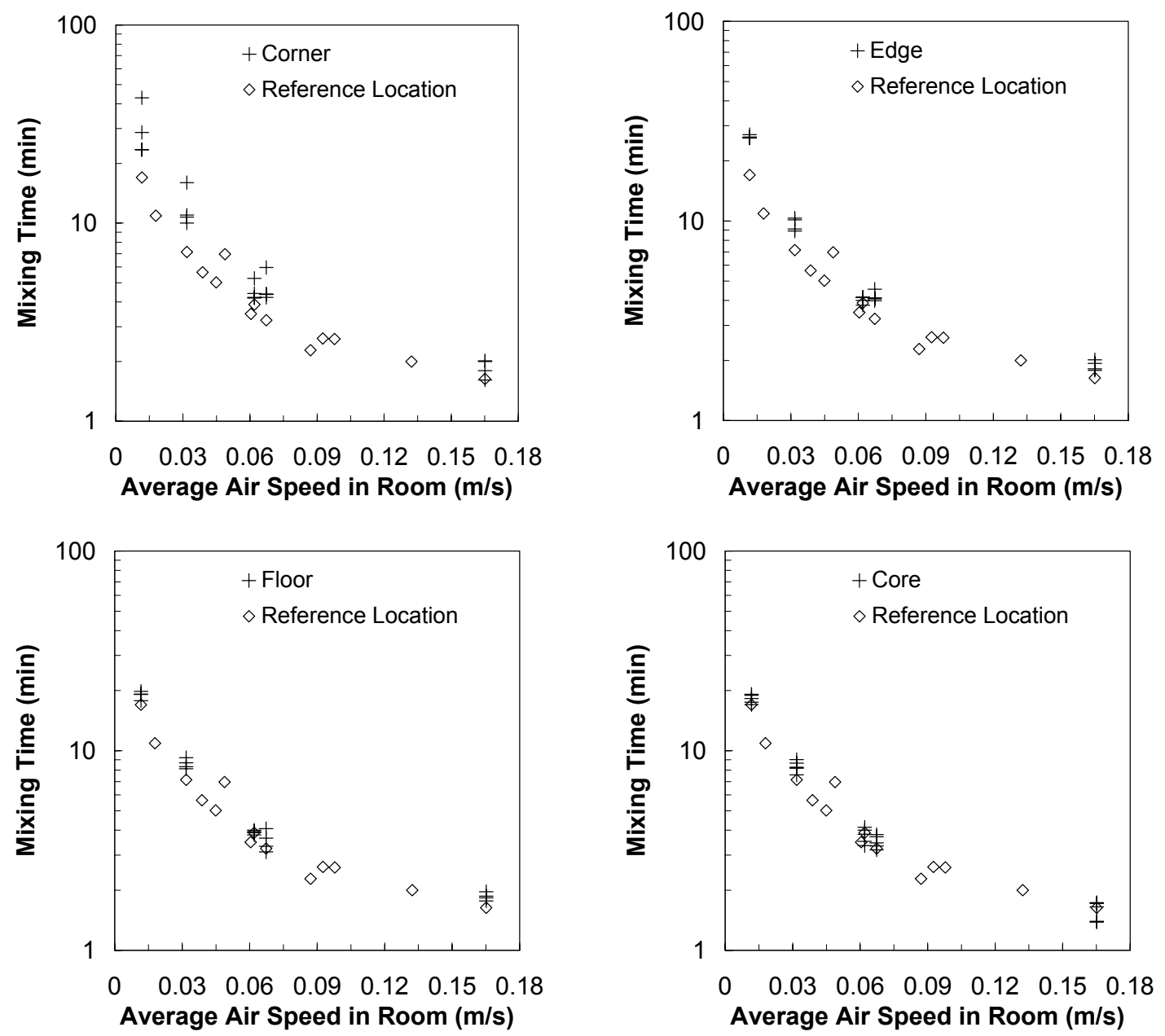

\section{Figure 5}




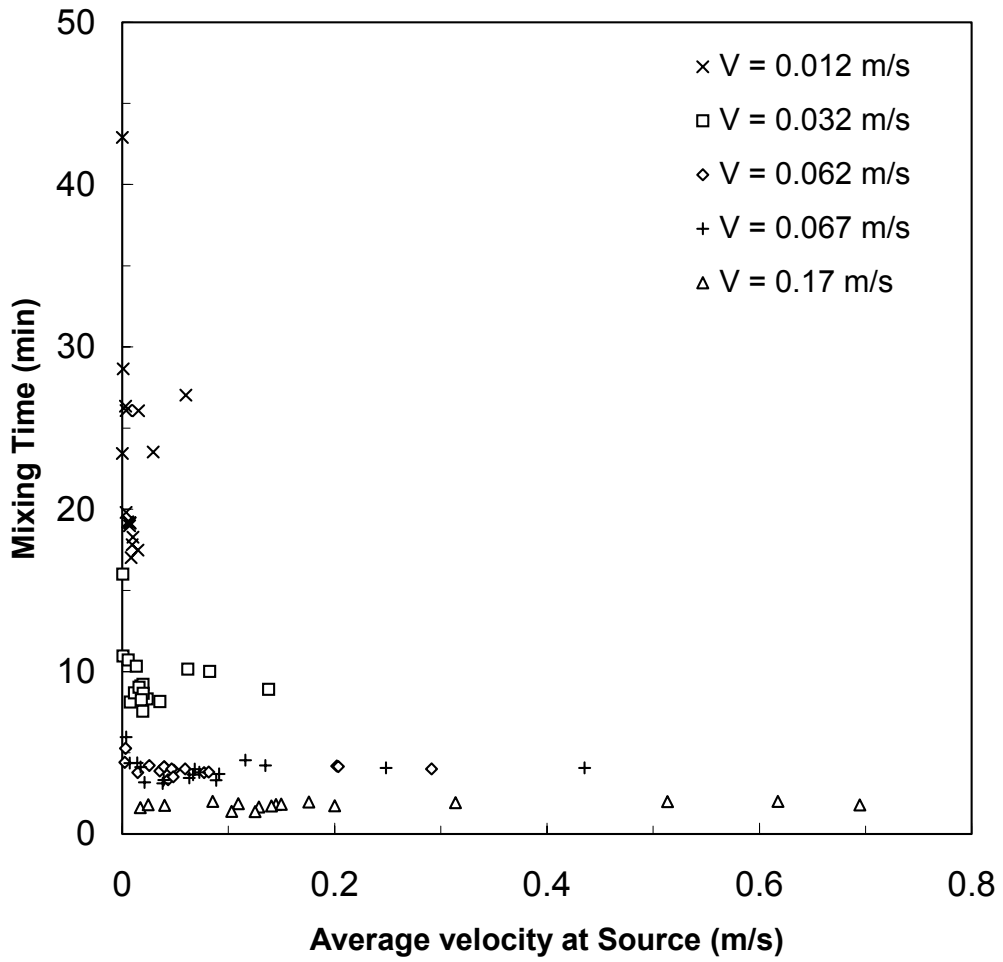

Figure 6 


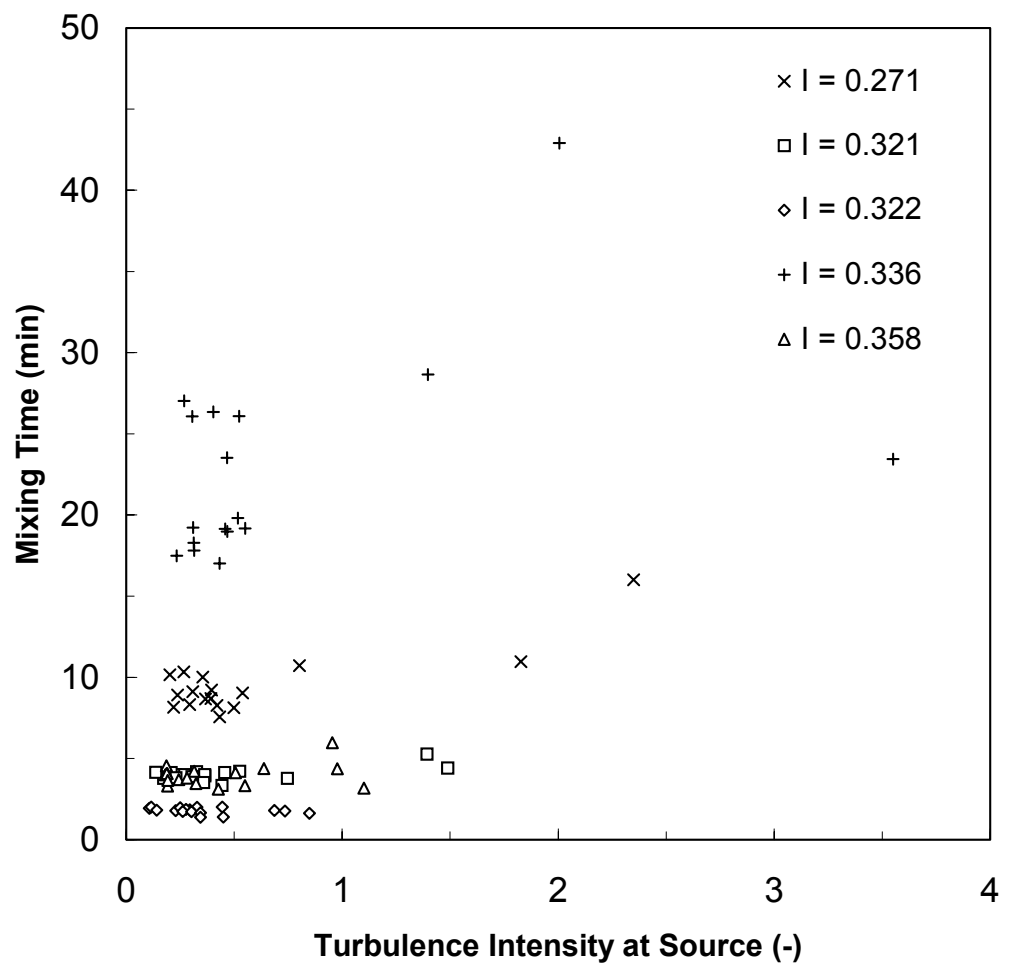

Figure 7 